\title{
Familiarizing Students with Definition of Lebesgue Outer Measure Using Mathematica: Some Examples of Calculation Directly from Its Definition
}

\author{
Włodzimierz Wojas • Jan Krupa • \\ Jarosław Bojarski
}

Received: 30 November 2018 / Revised: 10 April 2019 / Accepted: 12 April 2019 / Published online: 18 December 2019

(C) The Author(s) 2019

\begin{abstract}
In this paper we present some examples of calculation the Lebesgue outer measure of some subsets of $\mathbb{R}^{2}$ directly from definition 1 . We will consider the following subsets of $\mathbb{R}^{2}:\left\{(x, y) \in \mathbb{R}^{2}: 0 \leq y \leq x^{2}, x \in[0,1]\right\}$, $\left\{(x, y) \in \mathbb{R}^{2}: 0 \leq y \leq \exp (-x), x \geq 0\right\},\left\{(x, y) \in \mathbb{R}^{2}: \ln x \leq y \leq 0, x \in(0,1]\right\},\left\{(x, y) \in \mathbb{R}^{2}: 0 \leq\right.$ $y \leq 1 / x, x \geq 1\},\left\{(x, y) \in \mathbb{R}^{2}: 0 \leq y \leq \sin x, x \in[0, \pi / 2]\right\},\left\{(x, y) \in \mathbb{R}^{2}: 0 \leq y \leq \exp (x), x \in[0,1]\right\}$, $\left\{(x, y) \in \mathbb{R}^{2}: 0 \leq y \leq \ln \left(1-2 r \cos x+r^{2}\right), x \in[0, \pi]\right\}, r>1$ and some others. We could not find any analogical examples in available literature (except for rectangle and countable sets), so this paper is an attempt to fill this gap. We calculate sums, limits and plot graphs and dynamic plots of needed sets and unions of rectangles sums of which volumes approximate Lebesgue outer measure of the sets, using Mathematica. We also show how to calculate the needed sums and limits by hand (without CAS). The title of this paper is very similar to the title of author's article (Wojas and Krupa in Math Comput Sci 11:363-381, 2017) which deals with definition of Lebesgue integral but this paper deals with definition of Lebesgue outer measure instead.
\end{abstract}

Keywords Higher education $\cdot$ Lebesgue measure $\cdot$ Application of CAS $\cdot$ Mathematica $\cdot$ Mathematical didactics

Mathematics Subject Classification 97R20 -28A12 - 97B40

Electronic Supplementary material The online version of this article (https://doi.org/10.1007/s11786-019-00435-2) contains supplementary material, which is available to authorized users.

W. Wojas · J. Krupa $(\bowtie) \cdot$ J. Bojarski

Department of Applied Mathematics, Warsaw University of Life Sciences (SGGW), ul. Nowoursynowska 159, 02-776 Warsaw, Poland

e-mail: jan_krupa@sggw.pl; krupa1024@wp.pl

W. Wojas

e-mail: wlodzimierz_wojas@sggw.pl

J. Bojarski

e-mail: jaroslaw_bojarski@sggw.pl 
"Young man, in mathematics you don't understand things. You just get used to them"

John von Neumann

"I hear and I forget. I see and I remember. I do and I understand"

Chinese Quote

\section{Introduction}

The following definitions and lemmas we will use in our paper (compare [2,9]):

Rectangles. A closed rectangle $R$ in $\mathbb{R}^{d}$ is given by the product of $d$ one-dimensional closed and bounded intervals: $R=\left[a_{1}, b_{1}\right] \times\left[a_{2}, b_{2}\right] \times \cdots \times\left[a_{d}, b_{d}\right]$, where $a_{j} \leq b_{j}$ are real numbers, $j=1,2, \ldots, d$. In other words, we have $R=\left\{\left(x_{1}, \ldots, x_{d}\right) \in \mathbb{R}^{d}: a_{j} \leq x_{j} \leq b_{j}\right.$ for all $\left.j=1,2, \ldots, d\right\}$. We remark that in our definition, a rectangle is closed and has sides parallel to the coordinate axis. In $\mathbb{R}$, the rectangles are precisely the closed and bounded intervals, while in $\mathbb{R}^{2}$ they are the usual four-sided rectangles. In $\mathbb{R}^{3}$ they are the closed rectangular parallelepipeds.

An open rectangle is the product of open intervals, and the interior of the rectangle $R$ is then $\left(a_{1}, b_{1}\right) \times\left(a_{2}, b_{2}\right) \times \cdots \times\left(a_{d}, b_{d}\right)$.

We say that the lengths of the sides of the rectangle $R$ (open or closed) are $b_{1}-a_{1}, \ldots, b_{d}-a_{d}$. The volume of the rectangle $R$ (open or closed) is denoted by vol $(R)$, and is defined to be vol $(R)=\left(b_{1}-a_{1}\right) \cdots\left(b_{d}-a_{d}\right)$.

A union of rectangles is said to be almost disjoint if the interiors of the rectangles are disjoint. In this paper, coverings by rectangles play a major role, so we isolate here five important lemmas.

Lemma 1.1 If a closed rectangle is the almost disjoint union of finitely many other closed rectangles, say $R=$ $\bigcup_{k=1}^{N} R_{k}$, then $\operatorname{vol}(R)=\sum_{k=1}^{N} \operatorname{vol}\left(R_{k}\right)$.

Lemma 1.2 If $R, R_{1}, \ldots, R_{N}$ are closed rectangles, and $R \subset \bigcup_{k=1}^{N} R_{k}$, then

$\operatorname{vol}(R) \leq \sum_{k=1}^{N} \operatorname{vol}\left(R_{k}\right)$

Proof of the Lemmas 1.1, 1.2 can be found in [5,9,10].

Lemma 1.3 If $R_{1}, \ldots, R_{N}$ are almost disjoint closed rectangles, $Q_{1}, \ldots, Q_{M}$ are some other almost disjoint closed rectangles and $\bigcup_{k=1}^{N} R_{k} \subset \bigcup_{k=1}^{M} Q_{k}$, then $\sum_{k=1}^{N} \operatorname{vol}\left(R_{k}\right) \leq \sum_{k=1}^{M} \operatorname{vol}\left(Q_{k}\right)$.

The proof of 1.3 can be found in [5].

The ideas of the presented below proofs of lemmas are well known but we could not find the sources of the proofs.

Lemma 1.4 If $R_{1}, \ldots, R_{N}$ are almost disjoint closed rectangles, $Q_{1}, \ldots, Q_{M}$ are some closed rectangles and $\bigcup_{k=1}^{N} R_{k} \subset \bigcup_{k=1}^{M} Q_{k}$, then $\sum_{k=1}^{N} \operatorname{vol}\left(R_{k}\right) \leq \sum_{k=1}^{M} \operatorname{vol}\left(Q_{k}\right)$.

Proof Because $\bigcup_{k=1}^{N} R_{k} \subset \bigcup_{k=1}^{M} Q_{k}$ and $R_{1}, \ldots, R_{N}$ are almost disjoint we have:

$$
\begin{aligned}
& R_{i} \subset \bigcup_{j=1}^{M} R_{i} \cap Q_{j} \text { for } i=1,2, \ldots N \text { and } \\
& Q_{j} \supset \bigcup_{i=1}^{N} R_{i} \cap Q_{j} \text { for } j=1,2, \ldots M .
\end{aligned}
$$


By Lemma 1.2 we have:

$\operatorname{vol}\left(R_{i}\right) \leq \sum_{j=1}^{M} \operatorname{vol}\left(R_{i} \cap Q_{j}\right)$ for $i=1,2, \ldots N$

We see that for each $j=1,2, \ldots M$ the set $\left\{R_{i} \cap Q_{j}: i=1,2, \ldots n\right\}$ is collection of almost disjoint rectangles so by Lemma 1.3 we have:

$\operatorname{vol}\left(Q_{j}\right) \geq \sum_{i=1}^{N} \operatorname{vol}\left(R_{i} \cap Q_{j}\right)$ for $j=1,2, \ldots M$

Finally from Eqs. 1.1 and 1.2 we have:

$\sum_{i=1}^{N} \operatorname{vol}\left(R_{i}\right) \leq \sum_{i=1}^{N} \sum_{j=1}^{M} \operatorname{vol}\left(R_{i} \cap Q_{j}\right)=\sum_{j=1}^{M} \sum_{i=1}^{N} \operatorname{vol}\left(R_{i} \cap Q_{j}\right) \leq \sum_{j=1}^{M} \operatorname{vol}\left(Q_{j}\right)$

In the last proof instead of Lemma 1.3 we could use simpler lemma: If a closed rectangle $R$ contains the almost disjoint union of finitely many other closed rectangles, say $\bigcup_{k=1}^{N} R_{k} \subset R$, then $\sum_{k=1}^{N} \operatorname{vol}\left(R_{k}\right) \leq \operatorname{vol}(R)$ which can be proved by modifying the proof of the Lemma 1.1 from [9] or its proof can be found in [10]. The Lemma 1.4 could also be proved directly by modifying the proof of the Lemma 1.3 from [5].

Applying the previous Lemma 1.4 we can prove (see [9]):

Lemma 1.5 If $R_{1}, \ldots, R_{N}$ are almost disjoint closed rectangles, $Q_{k}, k=1,2, \ldots$ are some closed rectangles and $\bigcup_{k=1}^{N} R_{k} \subset \bigcup_{k=1}^{\infty} Q_{k}$, then $\sum_{k=1}^{N} \operatorname{vol}\left(R_{k}\right) \leq \sum_{k=1}^{\infty} \operatorname{vol}\left(Q_{k}\right)$.

Proof Let $A=\bigcup_{k=1}^{N} R_{k}$. For a fixed $\varepsilon>0$ we choose for each $k$ an open rectangle $P_{k}$ which contains $Q_{k}$ $\left(Q_{k} \subset P_{k}\right)$, and such that $\operatorname{vol}\left(P_{k}\right) \leq(1+\varepsilon) \operatorname{vol}\left(Q_{k}\right)$. From the open covering $\bigcup_{k=1}^{\infty} P_{k}$ of the compact set $A$, we may select a finite subcovering which, after possibly renumbering the rectangles, we may write as $A \subset \bigcup_{k=1}^{M} P_{k}$. Taking the closure of the rectangles $P_{k}$, we may apply Lemma 1.4 to conclude that for any $\varepsilon$ we have:

$\sum_{k=1}^{N} \operatorname{vol}\left(R_{k}\right) \leq \sum_{k=1}^{M} \operatorname{vol}\left(P_{k}\right) \leq(1+\varepsilon) \sum_{k=1}^{M} \operatorname{vol}\left(Q_{k}\right) \leq(1+\varepsilon) \sum_{k=1}^{\infty} \operatorname{vol}\left(Q_{k}\right)$. Since $\varepsilon$ is arbitrary, we find that $\sum_{k=1}^{N} \operatorname{vol}\left(R_{k}\right) \leq \sum_{k=1}^{\infty} \operatorname{vol}\left(Q_{k}\right)$.

Definition 1 (Lebesgue outer measure) (see $[1,2,4,6,8,9]$ ) The outer measure $m^{*}$ for any $A \subset \mathbb{R}^{d}$ is defined by the following formula:

$m^{*}(A)=\inf \left\{\sum_{j=1}^{\infty} \operatorname{vol}\left(R_{j}\right): A \subset \bigcup_{j=1}^{\infty} R_{j}, \quad R_{j}\right.$ is closed rectangle in $\left.\mathbb{R}^{d}, j \in \mathbb{N}\right\}$.

Definition 2 (Lebesgue measure) (see $[1,2,4,6,8,9]$ ) Let $\left(\mathbb{R}^{d}, \mathfrak{M}, m\right)$ be measure space, where $\mathfrak{M}$ is $\sigma$-algebra of Lebesgue measurable subsets in $\mathbb{R}^{d}$, and $m$-Lebesgue measure on $\mathbb{R}^{d}$. The measure $m$ for any $A \in \mathfrak{M}$ is defined by the formula $1.3\left(m(A)=m^{*}(A)\right)$.

2 Example 1: $A=\left\{(x, y) \in \mathbb{R}^{2}: 0 \leq y \leq x^{2}, 0 \leq x \leq 1\right\}$

Let $A$ be the set in $\mathbb{R}^{2}$ bounded by curves: $y=x^{2}, y=0, x=1$, which means that $A=\left\{(x, y) \in \mathbb{R}^{2}: 0 \leq\right.$ $\left.y \leq x^{2}, 0 \leq x \leq 1\right\}$. Let us calculate Lebesgue outer measure of $A$ using only formula (1.3) from Definition 1 and Lemma 1.5 . 
For $n \in \mathbb{N}$ define: $\bar{R}_{j}^{n}=\left[\frac{j-1}{n}, \frac{j}{n}\right] \times\left[0, \frac{j^{2}}{n^{2}}\right], j=1,2, \ldots n$ and $\underline{R}_{j}^{n}=\left[\frac{j-1}{n}, \frac{j}{n}\right] \times\left[0, \frac{(j-1)^{2}}{n^{2}}\right], j=1,2, \ldots n$.

Step 1. $\sum_{j=1}^{n} \operatorname{vol}\left(\bar{R}_{j}^{n}\right)=\sum_{j=1}^{n} \frac{1}{n} \frac{j^{2}}{n^{2}}=\frac{1}{n^{3}} \sum_{j=1}^{n} j^{2}=\frac{1}{n^{3}} \frac{n(n+1)(2 n+1)}{6}=\frac{1}{n^{2}} \frac{(n+1)(2 n+1)}{6} \rightarrow \frac{1}{3}$.

We can check our hand calculation using Wolfram Mathematica (see [7,12]) :

Listing 1 Mathematica code:

$$
\begin{aligned}
& \text { In[1]:= Simplify }\left[\frac{1}{n^{3}} \sum_{j=1}^{n} j^{2}\right] \\
& \text { Out[1] }=\frac{1}{n^{2}} \frac{(n+1)(2 n+1)}{6} \\
& \operatorname{In}[2]:=\operatorname{Limit}[\%, n \rightarrow \infty] \\
& \text { Out[2]= } \frac{1}{3}
\end{aligned}
$$

Hence because $A \subset \bigcup_{j=1}^{n} \bar{R}_{j}^{n}$, we have:

$$
\begin{aligned}
m^{*}(A) & =\inf \left\{\sum_{j=1}^{\infty} \operatorname{vol}\left(R_{j}\right): A \subset \bigcup_{j=1}^{\infty} R_{j}, \quad R_{j} \text { is closed rectangle in } \mathbb{R}^{2}, j \in \mathbb{N}\right\} \\
& \leq \inf \left\{\sum_{j=1}^{n} \operatorname{vol}\left(\bar{R}_{j}^{n}\right): n \in \mathbb{N}\right\} \leq \frac{1}{3} .
\end{aligned}
$$

Step 2. Note that:

$$
\begin{aligned}
\sum_{j=1}^{n} \operatorname{vol}\left(\underline{R}_{j}^{n}\right) & =\sum_{j=1}^{n} \frac{1}{n} \frac{(j-1)^{2}}{n^{2}}=\frac{1}{n^{3}} \sum_{j=1}^{n-1} j^{2} \\
& =\frac{1}{n^{3}} \frac{(n-1) n(2 n-1)}{6}=\frac{1}{n^{2}} \frac{(n-1)(2 n-1)}{6} \rightarrow \frac{1}{3} .
\end{aligned}
$$

We can check our hand calculation using Wolfram Mathematica:

Listing 2 Mathematica code:

$$
\begin{aligned}
& \operatorname{In}[3]:=\text { Simplify }\left[\frac{1}{n^{3}} \sum_{j=1}^{n-1} j^{2}\right] \\
& \text { Out[3] }=\frac{1}{n^{3}} \frac{(n-1) n(2 n-1)}{6} \\
& \operatorname{In}[4]:=\operatorname{Limit}[\%, n \rightarrow \infty] \\
& \text { Out[4]= } \frac{1}{3}
\end{aligned}
$$

The dynamic versions of the Figs. 1 and 2 can be found in the Electronic supplementary material. 
Fig. 1 Approximation of Lebesgue outer measure of $A$ by rectangles $\bigcup_{j=1}^{n} \underline{R}_{j}^{n}$ for Example 1

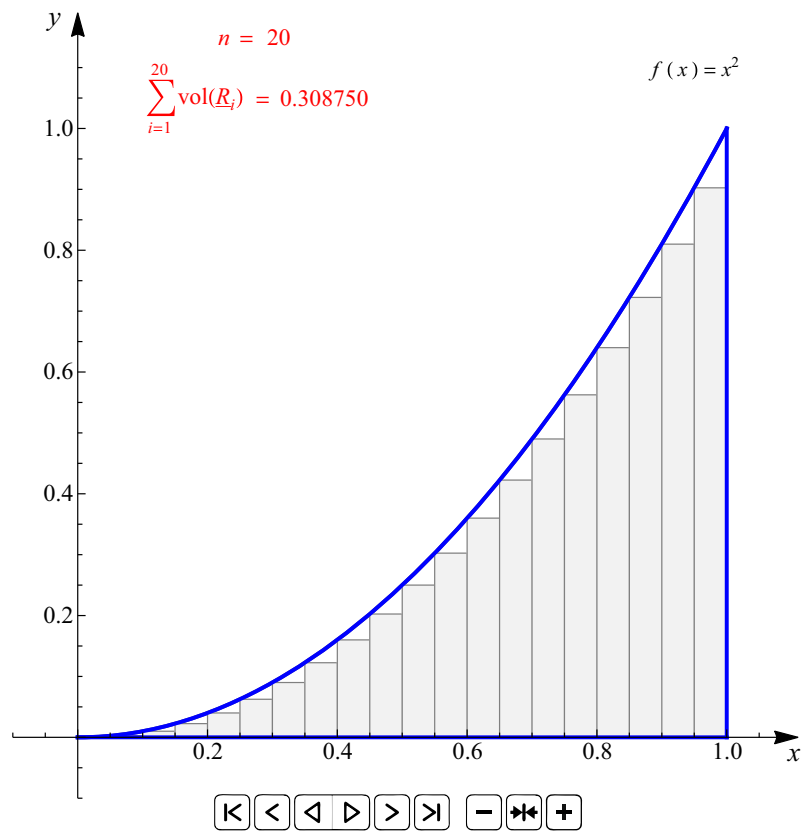

Fig. 2 Approximation of Lebesgue outer measure of $A$ by rectangles $\bigcup_{j=1}^{n} \bar{R}_{j}^{n}$ for Example 1

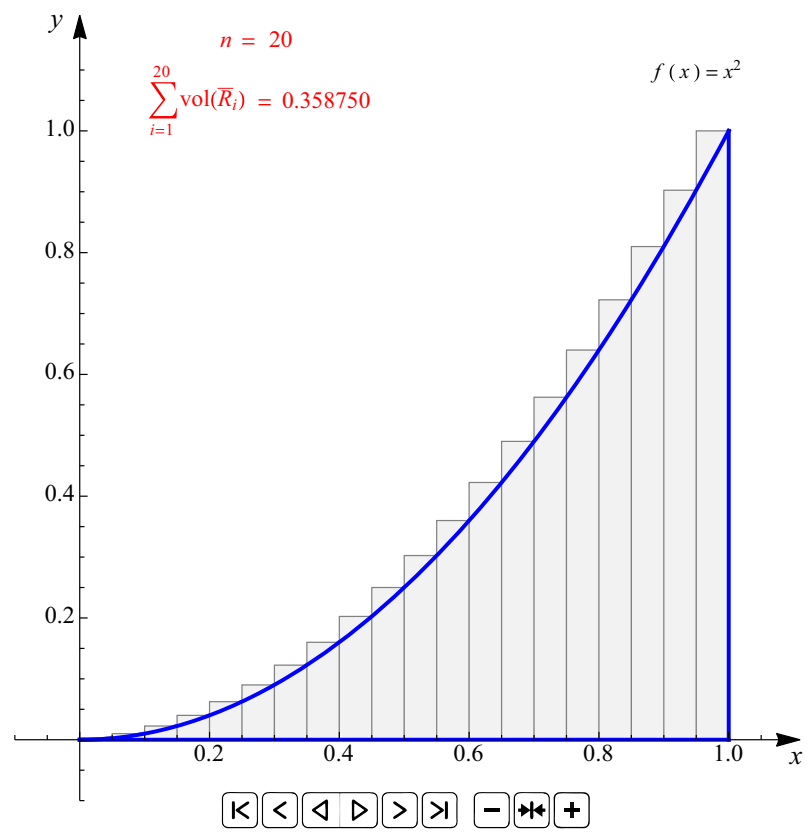

We consider an arbitrary countable covering $A \subset \bigcup_{j=1}^{\infty} R_{j}$ by closed rectangles.

Hence because $\bigcup_{j=1}^{n} \underline{R}_{j}^{n} \subset A \subset \bigcup_{j=1}^{\infty} R_{j}$ from Lemma 1.5 we have that for any $n$ : $\sum_{j=1}^{n} \operatorname{vol}\left(\underline{R}_{j}^{n}\right) \leq \sum_{j=1}^{\infty} \operatorname{vol}\left(R_{j}\right)$. 
Applying the formula 2.2 we have: $\sum_{j=1}^{\infty} \operatorname{vol}\left(R_{j}\right) \geq \lim _{n \rightarrow \infty} \sum_{j=1}^{n} \operatorname{vol}\left(\underline{R}_{j}^{n}\right)=\frac{1}{3}$. Consequently,

$m^{*}(A)=\inf \left\{\sum_{j=1}^{\infty} \operatorname{vol}\left(R_{j}\right): A \subset \bigcup_{j=1}^{\infty} R_{j}, \quad R_{j}\right.$ is closed rectangle in $\left.\mathbb{R}^{2}, j \in \mathbb{N}\right\} \geq \frac{1}{3}$

From inequalities (2.1) and (2.3) we have $m^{*}(A)=\frac{1}{3}$ directly from formula (1.3) from Definition 1 . The set $A$ is Lebesgue measurable because $A$ is closed, so $m(A)=\frac{1}{3}$.

\section{Example 2: $A=\left\{(x, y) \in \mathbb{R}^{2}: 0 \leq y \leq \exp (-x), x \geq 0\right\}$}

Let $A$ be the set in $\mathbb{R}^{2}$ bounded by curves: $y=\exp (-x), y=0$ for $x \in[0, \infty)$, which means that $A=\{(x, y) \in$ $\left.\mathbb{R}^{2}: 0 \leq y \leq \exp (-x), x \geq 0\right\}$. Let us calculate Lebesgue outer measure of $A$ using only formula (1.3) from Definition 1 and Lemma 1.5.

For $n \in \mathbb{N}$ define: $\bar{R}_{j k}^{n}=\left[k+\frac{j}{2^{n}}, k+\frac{j+1}{2^{n}}\right] \times\left[0, \exp \left(-k-\frac{j}{2^{n}}\right)\right], j=0,1,2, \ldots, 2^{n}-1, k=0,1,2, \ldots \infty$, and $\underline{R}_{j}^{n}=\left[\frac{j}{2^{n}}, \frac{j+1}{2^{n}}\right] \times\left[0, \exp \left(-\frac{j+1}{2^{n}}\right)\right], j=0,1,2, \ldots n 2^{n}-1$.

Step 1. Using Wolfram Mathematica:

Listing 3 Mathematica code:

$$
\begin{aligned}
& \operatorname{In}[5]:=\text { Simplify }\left[\sum_{j=0}^{2^{n}-1} \sum_{k=0}^{\infty} \frac{1}{2^{n}} \operatorname{Exp}\left[\left(-k-\frac{j}{2^{n}}\right)\right]\right] \\
& \text { Out[5] }=\frac{2^{-n} e^{2^{-n}}}{e^{2^{-n}}-1} \\
& \operatorname{In}[6]:=\operatorname{Limit}[\%, n \rightarrow \infty] \\
& \text { Out[6] }=1
\end{aligned}
$$

we get:

$$
\sum_{j=0}^{2^{n}-1} \sum_{k=0}^{\infty} \operatorname{vol}\left(\bar{R}_{j k}^{n}\right)=\sum_{j=0}^{2^{n}-1} \sum_{k=0}^{\infty} \frac{1}{2^{n}} \exp \left(-k-\frac{j}{2^{n}}\right)=\frac{2^{-n} e^{2^{-n}}}{e^{2^{-n}}-1} \rightarrow 1
$$

Hence because $A \subset \bigcup_{j=0}^{2^{n}-1} \bigcup_{k=0}^{\infty} \bar{R}_{j k}^{n}$, we have:

$$
\begin{aligned}
m^{*}(A) & =\inf \left\{\sum_{j=1}^{\infty} \operatorname{vol}\left(R_{j}\right): A \subset \bigcup_{j=1}^{\infty} R_{j}, \quad R_{j} \text { is closed rectangle in } \mathbb{R}^{2}, j \in \mathbb{N}\right\} \\
& \leq \inf \left\{\sum_{j=0}^{2^{n}-1} \sum_{k=0}^{\infty} \operatorname{vol}\left(\bar{R}_{j k}^{n}\right): n \in \mathbb{N}\right\} \leq 1 .
\end{aligned}
$$

Step 2. Using Wolfram Mathematica: 
Listing 4 Mathematica code:

$$
\begin{aligned}
& \text { In[7]:= Simplify }\left[\sum_{j=0}^{n 2^{n}-1} \frac{1}{2^{n}} \operatorname{Exp}\left[-\frac{j+1}{2^{n}}\right]\right] \\
& \text { Out[7] }=\frac{(2 e)^{-n}\left(e^{n}-1\right)}{e^{2^{-n}}-1} \\
& \operatorname{In}[8]:=\operatorname{Limit}[\%, n \rightarrow \infty] \\
& \text { Out[8]= }
\end{aligned}
$$

We get that:

$$
\sum_{j=0}^{n 2^{n}-1} \operatorname{vol}\left(\underline{R}_{j}^{n}\right)=\sum_{j=0}^{n 2^{n}-1} \frac{1}{2^{n}} \exp \left(-\frac{j+1}{2^{n}}\right)=\frac{(2 e)^{-n}\left(e^{n}-1\right)}{e^{2^{-n}}-1} \rightarrow 1
$$

Of course, we could use the following formulae: $\sum_{k=0}^{n} q^{k}=\frac{1-q^{n+1}}{1-q}(q \neq 1)$ and $\lim _{x \rightarrow 0} \frac{\exp x-1}{x}=1$ instead of the code in Listings 3 and 4 to get the results in formulae (3.1) and (3.3).

We consider an arbitrary countable covering $A \subset \bigcup_{j=1}^{\infty} R_{j}$ by closed rectangles.

Hence because $\bigcup_{j=0}^{n 2^{n}-1} \underline{R}_{j}^{n} \subset A \subset \bigcup_{j=1}^{\infty} R_{j}$ from Lemma 1.5 we have that for any $n$ :

$\sum_{j=0}^{n 2^{n}-1} \operatorname{vol}\left(\underline{R}_{j}^{n}\right) \leq \sum_{j=1}^{\infty} \operatorname{vol}\left(R_{j}\right)$.

Applying the formula 3.3 we have: $\sum_{j=1}^{\infty} \operatorname{vol}\left(R_{j}\right) \geq \lim _{n \rightarrow \infty} \sum_{j=0}^{n 2^{n}-1} \operatorname{vol}\left(\underline{R}_{j}^{n}\right)=1$. Consequently,

$$
m^{*}(A)=\inf \left\{\sum_{j=1}^{\infty} \operatorname{vol}\left(R_{j}\right): A \subset \bigcup_{j=1}^{\infty} R_{j}, \quad R_{j} \text { is closed rectangle in } \mathbb{R}^{2}, j \in \mathbb{N}\right\} \geq 1
$$

From inequalities (3.2) and (3.4) we have $m^{*}(A)=1$ directly from formula (1.3) from Definition 1 . The set $A$ is Lebesgue measurable because $A$ is closed, so $m(A)=1$.

The dynamic versions of the Figs. 3 and 4 can be found in the Electronic supplementary material.

4 Example 3: $A=\left\{(x, y) \in \mathbb{R}^{2}: \ln x \leq y \leq 0, x \in(0,1]\right\}$

Let $A$ be the set in $\mathbb{R}^{2}$ bounded by curves: $y=\ln x, y=0$ for $x \in(0,1]$, which means that $A=\left\{(x, y) \in \mathbb{R}^{2}\right.$ : $\ln x \leq y \leq 0, x \in(0,1]\}$. Let us calculate Lebesgue outer measure of $A$ using only formula (1.3) from Definition 1 and Lemma 1.5.

For $n \in \mathbb{N}$ define: $\bar{R}_{j}^{n}=\left[\frac{j}{2^{n}}, \frac{j+1}{2^{n}}\right] \times\left[\ln \frac{j}{2^{n}}, 0\right], j=1,2, \ldots, 2^{n}-1, \bar{Q}_{k}^{n}=\left[\frac{1}{2^{k+1}}, \frac{1}{2^{k}}\right] \times\left[\ln \frac{1}{2^{k+1}}, 0\right], k=$ $n, n+1, \ldots, \infty$, and $\underline{R}_{j}^{n}=\left[\frac{j}{n}, \frac{j+1}{n}\right] \times\left[\ln \frac{j+1}{n}, 0\right], j=1,2, \ldots, n-1$.

\section{Step 1.}

We can see that

$$
\sum_{k=n}^{\infty} \operatorname{vol}\left(\bar{Q}_{k}^{n}\right)=-\sum_{k=n}^{\infty} \frac{1}{2^{k+1}} \ln \left(\frac{1}{2^{k+1}}\right)=\frac{(n+2)}{2^{n}} \ln 2 \rightarrow 0 \text {. }
$$


Fig. 3 Approximation of Lebesgue outer measure of $A$ by rectangles $\bigcup_{j=0}^{n 2^{n}-1} \underline{R}_{j}^{n}$ for Example 2

Fig. 4 Approximation of Lebesgue outer measure of $A$ by rectangles $\bigcup_{j=0}^{2^{n}-1} \bigcup_{k=0}^{\infty} \bar{R}_{j k}^{n}$ for Example 2
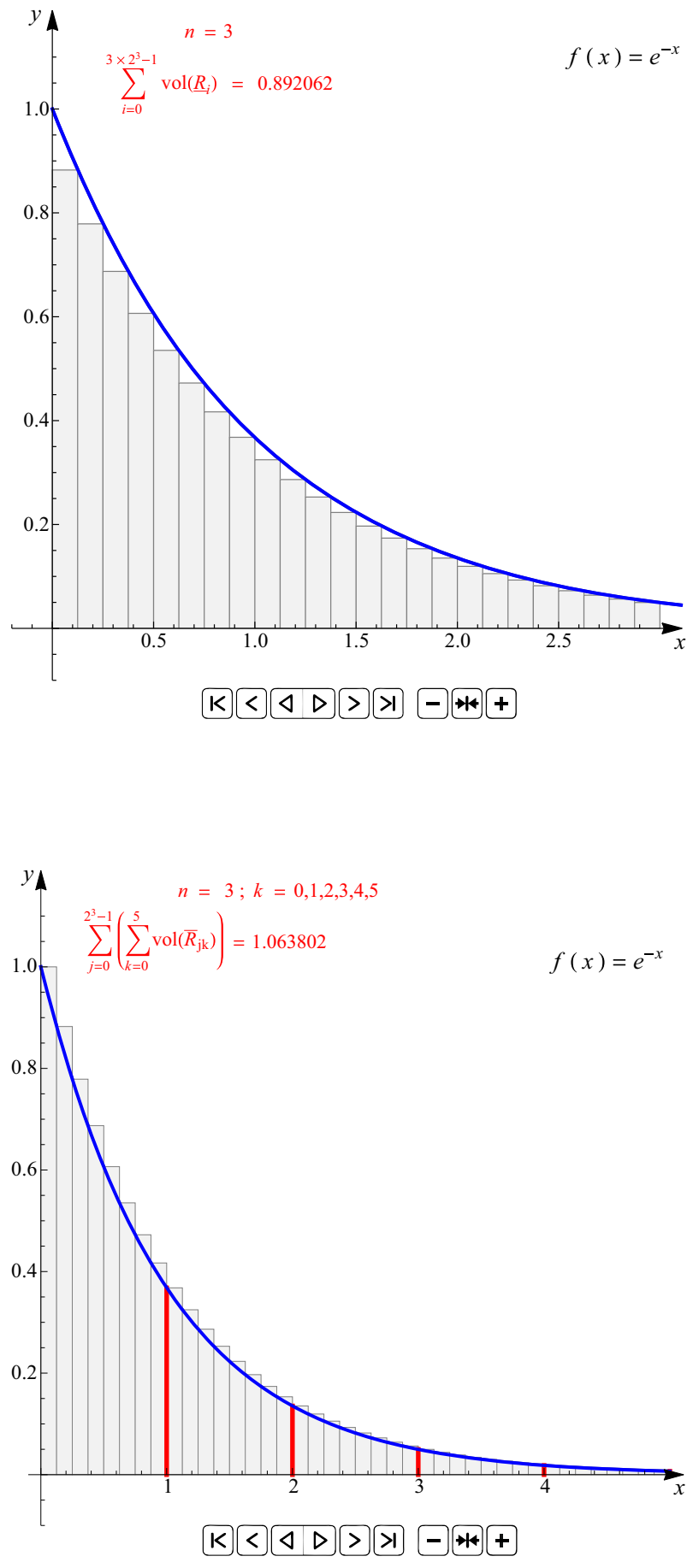

$\sum_{j=1}^{2^{n}-1} \operatorname{vol}\left(\bar{R}_{j}^{n}\right)=-\frac{1}{2^{n}} \sum_{j=1}^{2^{n}-1} \ln \left(\frac{j}{2^{n}}\right)=-\ln \frac{\sqrt[2^{n}]{\left(2^{n}\right) !}}{2^{n}}+\ln \sqrt[2^{n}]{2^{n}} \rightarrow 1$ 
because from calculus we know that $\frac{\sqrt[n]{n !}}{n} \rightarrow 1 / e$. Hence, because $A \subset \bigcup_{k=n}^{\infty} \bar{Q}_{k}^{n} \cup \bigcup_{j=1}^{2^{n}-1} \bar{R}_{j}^{n}$, we have:

$$
\begin{aligned}
m^{*}(A) & =\inf \left\{\sum_{j=1}^{\infty} \operatorname{vol}\left(R_{j}\right): A \subset \bigcup_{j=1}^{\infty} R_{j}, \quad R_{j} \text { is closed rectangle in } \mathbb{R}^{2}, j \in \mathbb{N}\right\} \\
& \leq \inf \left\{\sum_{k=n}^{\infty} \bar{Q}_{k}^{n}+\sum_{j=1}^{2^{n}-1} \operatorname{vol}\left(\bar{R}_{j}^{n}\right): n \in \mathbb{N}\right\} \leq 1 .
\end{aligned}
$$

\section{Step 2.}

We get that:

$$
\sum_{j=1}^{n-1} \operatorname{vol}\left(\underline{R}_{j}^{n}\right)=-\frac{1}{n} \sum_{j=1}^{n-1} \ln \left(\frac{j+1}{n}\right)=-\ln \frac{\sqrt[n]{n !}}{n} \rightarrow 1
$$

We consider an arbitrary covering $A \subset \bigcup_{j=1}^{\infty} R_{j}$ by closed rectangles.

Hence, because $\bigcup_{j=1}^{n-1} \underline{R}_{j}^{n} \subset A \subset \bigcup_{j=1}^{\infty} R_{j}$ from Lemma 1.5 we have that for any $n$ :

$$
\sum_{j=1}^{n-1} \operatorname{vol}\left(\underline{R}_{j}^{n}\right) \leq \sum_{j=1}^{\infty} \operatorname{vol}\left(R_{j}\right) \text {. }
$$

Applying the formula 4.4 we have: $\sum_{j=1}^{\infty} \operatorname{vol}\left(R_{j}\right) \geq \lim _{n \rightarrow \infty} \sum_{j=1}^{n-1} \operatorname{vol}\left(\underline{R}_{j}^{n}\right)=1$. Consequently,

$$
m^{*}(A)=\inf \left\{\sum_{j=1}^{\infty} \operatorname{vol}\left(R_{j}\right): A \subset \bigcup_{j=1}^{\infty} R_{j}, \quad R_{j} \text { is closed rectangle in } \mathbb{R}^{2}, j \in \mathbb{N}\right\} \geq 1
$$

From inequalities (4.3) and (4.5) we have $m^{*}(A)=1$ directly from formula (1.3) from Definition 1 . The set $A$ is Lebesgue measurable because $A$ is Borel set, so $m(A)=1$.

Of course, we could use almost the same $\bar{R}_{j k}^{n}$ and $\underline{R}_{j}^{n}$ like in example 2 in Sect. 3.

5 Example 4: $A=\left\{(x, y) \in \mathbb{R}^{2}: 0 \leq y \leq 1 / x, x \geq 1\right\}$

Let $A$ be the set in $\mathbb{R}^{2}$ bounded by curves: $y=1 / x, y=0$ for $x \in[1, \infty)$, which means that $A=\left\{(x, y) \in \mathbb{R}^{2}\right.$ : $0 \leq y \leq 1 / x, x \geq 1\}$. Let us calculate Lebesgue outer measure of $A$ using only formula (1.3) from Definition 1 and Lemma 1.5.

For $n \in \mathbb{N}$ define:

$\underline{R}_{k}^{n}=[k, k+1] \times\left[0, \frac{1}{k+1}\right], k=1,2, \ldots n$.

We can see that

$$
\sum_{k=1}^{n} \operatorname{vol}\left(\underline{R}_{k}^{n}\right)=\sum_{k=1}^{n} \frac{1}{k+1} \rightarrow \infty
$$

We consider an arbitrary covering $A \subset \bigcup_{j=1}^{\infty} R_{j}$ by closed rectangles.

Hence, because $\bigcup_{k=1}^{n} \underline{R}_{k}^{n} \subset A \subset \bigcup_{j=1}^{\infty} R_{j}$ from Lemma 1.5 we have that for any $n$ :

$\sum_{k=1}^{n} \operatorname{vol}\left(\underline{R}_{k}^{n}\right) \leq \sum_{j=1}^{\infty} \operatorname{vol}\left(R_{j}\right)$. 
Applying the formula 5.1 we have: $\sum_{j=1}^{\infty} \operatorname{vol}\left(R_{j}\right) \geq \lim _{n \rightarrow \infty} \sum_{k=1}^{n} \operatorname{vol}\left(\underline{R}_{k}^{n}\right)=\infty$. Consequently,

$m^{*}(A)=\inf \left\{\sum_{j=1}^{\infty} \operatorname{vol}\left(R_{j}\right): A \subset \bigcup_{j=1}^{\infty} R_{j}, \quad R_{j}\right.$ is closed rectangle in $\left.\mathbb{R}^{2}, j \in \mathbb{N}\right\} \geq \infty$

From inequality (5.2) we have $m^{*}(A)=\infty$ directly from formula (1.3) from Definition 1 . The set $A$ is Lebesgue measurable because $A$ is closed, so $m(A)=\infty$.

Similarly, we can prove directly from formula (1.3) from Definition 1 that $m^{*}\left(\left\{(x, y) \in \mathbb{R}^{2}: 0 \leq y \leq 1 / x, x \in\right.\right.$ $(0,1]\})=\infty$.

6 Example 5: $A=\left\{(x, y) \in \mathbb{R}^{2}: 0 \leq y \leq f(x), 0 \leq x \leq 1\right\}$, where $f(x)$ is Function Defined in (6.1)

$f(x)=x \chi_{[0,1] \cap \mathbb{Q}}(x)= \begin{cases}x & \text { if } x \in[0,1] \cap \mathbb{Q}, \\ 0 & \text { if } x \in[0,1] \backslash \mathbb{Q} .\end{cases}$

Let $A=\left\{(x, y) \in \mathbb{R}^{2}: 0 \leq y \leq f(x), 0 \leq x \leq 1\right\}$, where $f(x)$ is function defined in (6.1). Let us calculate Lebesgue outer measure of $A$ using only formula (1.3) from definition 1.

Let $r_{i}, i=1,2,3, \ldots$ be a sequence of all rational numbers from the interval $[0,1]$.

For $n \in \mathbb{N}$ define:

$R_{0}=[0,1] \times\{0\}, R_{i}=\left[r_{i}-\frac{1}{2^{n+i+1}}, r_{i}+\frac{1}{2^{n+i+1}}\right] \times[0,1]$ for $i=1,2,3, \ldots$

One can see that for $n \in \mathbb{N}$ we have:

$A \subset R_{0} \cup \bigcup_{i=1}^{\infty} R_{i}$

$\operatorname{vol}\left(R_{0}\right)+\sum_{i=1}^{\infty} \operatorname{vol}\left(R_{i}\right) \leq 0+\sum_{i=1}^{\infty} \frac{1}{2^{n+i}}=\frac{1}{2^{n}} \rightarrow 0 \quad$ as $n \rightarrow \infty$.

From (6.2), (6.3) and properties of the greatest lower bound we have $m^{*}(A)=0$ directly from formula (1.3) from Definition 1. The set $A$ is Lebesgue measurable because $m^{*}(A)=0$, so $m(A)=0$.

Similarly we can calculate Lebesgue outer measure of $A=\left\{(x, y) \in \mathbb{R}^{2}: 0 \leq y \leq g(x), 0 \leq x \leq 1\right\}$, where $g(x)$ is function defined in (6.4), using only formula (1.3) from Definition 1.

$g(x)= \begin{cases}1 & \text { if } x=0 \\ \frac{1}{q} & \text { if } x=\frac{p}{q} \in[0,1] \cap \mathbb{Q}, \frac{p}{q} \text { is in the lowest terms } \\ 0 & \text { if } x \in[0,1] \backslash \mathbb{Q} .\end{cases}$

7 Example 6: $A=\left\{(x, y) \in \mathbb{R}^{2}: 0 \leq y \leq f(x), x \in[0, \infty)\right\}$, where $f(x)$ is Function Defined in (7.1)

$f(x)=e^{x} \chi_{[0, \infty) \cap \mathbb{Q}}(x)= \begin{cases}e^{x} & \text { if } x \in[0, \infty) \cap \mathbb{Q}, \\ 0 & \text { if } x \in[0, \infty) \backslash \mathbb{Q} .\end{cases}$

Let $A=\left\{(x, y) \in \mathbb{R}^{2}: 0 \leq y \leq f(x), x \in[0, \infty)\right\}$, where $f(x)$ is function defined in (7.1). Let us calculate Lebesgue outer measure of $A$ using only formula (1.3) from Definition 1.

Let $r_{i}, i=0,1,2,3, \ldots$ be a sequence of all rational numbers from the interval $[0, \infty)$. 
For $n \in \mathbb{N}$ define:

$$
\begin{aligned}
& R_{i}^{n}=\left[r_{i}-\frac{1}{e^{r_{i}} 2^{n+3+i}}, r_{i}+\frac{1}{e^{r_{i}} 2^{n+3+i}}\right] \times\left[0, e^{r_{i}}\right] \text { for } i=0,1,2,3, \ldots \text { and } \\
& Q_{k}^{n}=[k, k+1] \times\left[0, \frac{1}{2^{n+k+2}}\right], \quad k=0,1,2, \ldots
\end{aligned}
$$

One can see that for $n \in \mathbb{N}$ we have:

$$
\begin{aligned}
& A \subset \bigcup_{k=0}^{\infty} Q_{k}^{n} \cup \bigcup_{i=0}^{\infty} R_{i}^{n} \\
& \sum_{k=0}^{\infty} \operatorname{vol}\left(Q_{k}^{n}\right)+\sum_{i=0}^{\infty} \operatorname{vol}\left(R_{i}^{n}\right)=\sum_{k=0}^{\infty} \frac{1}{2^{n+k+2}}+\sum_{i=0}^{\infty} \frac{1}{2^{n+i+2}}=\frac{1}{2^{n}} \rightarrow 0 \quad \text { as } n \rightarrow \infty .
\end{aligned}
$$

From (7.2), (7.3) and properties of the greatest lower bound we have $m^{*}(A)=0$ directly from formula (1.3) from Definition 1. The set $A$ is Lebesgue measurable because $m^{*}(A)=0$, so $m(A)=0$.

\section{Example 7: $A=[0,1] \times[0,1] \backslash \mathbb{Q} \times \mathbb{Q}$}

Let us calculate Lebesgue outer measure of $A$ using only formula (1.3) from Definition 1.

Let $r_{i}=\left(p_{i}, q_{i}\right) \in A, i=1,2,3, \ldots$ be a sequence of all points of $A$ with both rational coordinates.

Because $A \subset[0,1] \times[0,1]$ and $\operatorname{vol}([0,1] \times[0,1])=1$ we have that $m^{*}(A) \leq 1$.

Suppose there exist rectangles $R_{i} \subset \mathbb{R}^{2}$ such that $A \subset \bigcup_{i=1}^{\infty} R_{i}$ and $\sum_{i=1}^{\infty} \operatorname{vol}\left(R_{i}\right)=1-\varepsilon$ for some $\varepsilon>0$.

Let $Q_{i}=\left[p_{i}-\frac{\sqrt{\varepsilon}}{2^{(i+1) / 2+1}}, p_{i}+\frac{\sqrt{\varepsilon}}{2^{(i+1) / 2+1}}\right] \times\left[q_{i}-\frac{\sqrt{\varepsilon}}{2^{(i+1) / 2+1}}, q_{i}+\frac{\sqrt{\varepsilon}}{2^{(i+1) / 2+1}}\right]$ for $i=1,2,3, \ldots$

One can see that

$[0,1] \times[0,1] \subset \bigcup_{i=1}^{\infty} Q_{i} \cup \bigcup_{i=1}^{\infty} R_{i}$

and

$$
\sum_{i=1}^{\infty} \operatorname{vol}\left(Q_{i}\right)+\sum_{i=1}^{\infty} \operatorname{vol}\left(R_{i}\right)=\varepsilon \sum_{i=1}^{\infty} \frac{1}{2^{i+1}}+1-\varepsilon=1-\varepsilon / 2<1
$$

but $\operatorname{vol}([0,1] \times[0,1])=1$ which contradicts Lemma 1.5 so $m^{*}(A)=1$.

The set $A$ is Lebesgue measurable because $A$ is Borel set, so $m(A)=1$.

Similarly consider set $B=[0,1]^{d} \backslash \mathbb{Q}^{d}$. Because $B \subset[0,1]^{d}$ and $\operatorname{vol}\left([0,1]^{d}\right)=1$ we have that $m^{*}(B) \leq 1$.

Let $t_{i} \in[0,1]^{d} \cap \mathbb{Q}^{n}, i=1,2,3, \ldots$ be a sequence of all points of $B$ with all rational coordinates.

Suppose there exist rectangles $R_{i} \subset \mathbb{R}^{d}$ such that $B \subset \bigcup_{i=1}^{\infty} R_{i}$ and $\sum_{i=1}^{\infty} \operatorname{vol}\left(R_{i}\right)=1-\varepsilon$ for some $\varepsilon>0$.

For a fixed $\varepsilon>0$ we choose for each $i=1,2, \ldots$ a rectangle $P_{i}$ such that $t_{i} \in P_{i}$ and that $\operatorname{vol}\left(P_{i}\right)=\frac{\varepsilon}{2^{i+1}}$.

One can see that

$[0,1]^{d} \subset \bigcup_{i=1}^{\infty} P_{i} \cup \bigcup_{i=1}^{\infty} R_{i}$ 
and

$\sum_{i=1}^{\infty} \operatorname{vol}\left(P_{i}\right)+\sum_{i=1}^{\infty} \operatorname{vol}\left(R_{i}\right)=\varepsilon \sum_{i=1}^{\infty} \frac{1}{2^{i+1}}+1-\varepsilon=1-\varepsilon / 2<1$

but $\left.\operatorname{vol}\left([0,1]^{d}\right]\right)=1$ which contradicts Lemma 1.5 so $m^{*}(B)=1$.

The set $B$ is Lebesgue measurable because $B$ is Borel set, so $m(B)=1$.

9 Example 8: $A=\left\{(x, y) \in \mathbb{R}^{2}: 0 \leq y \leq \sin x, 0 \leq x \leq \pi / 2\right\}$

Let $A$ be the set in $\mathbb{R}^{2}$ bounded by curves: $y=\sin x, y=0$ for $x \in[0, \pi / 2]$, which means that $A=\{(x, y) \in$ $\left.\mathbb{R}^{2}: 0 \leq y \leq \sin x, 0 \leq x \leq \pi / 2\right\}$. Let us calculate Lebesgue outer measure of $A$ using only formula (1.3) from Definition 1 and Lemma 1.5 .

For $n \in \mathbb{N}$ define: $\bar{R}_{j}^{n}=\left[\frac{j \pi}{2 \cdot 2^{n}}, \frac{(j+1) \pi}{2 \cdot 2^{n}}\right] \times\left[0, \sin \frac{(j+1) \pi}{2 \cdot 2^{n}}\right], j=0,1,2, \ldots 2^{n}-1$ and $\underline{R}_{j}^{n}=\left[\frac{j \pi}{2 \cdot 2^{n}}, \frac{(j+1) \pi}{2 \cdot 2^{n}}\right] \times$ $\left[0, \sin \frac{j \pi}{2 \cdot 2^{n}}\right], j=0,1,2, \ldots 2^{n}-1$.

Step 1. Using Wolfram Mathematica:

Listing 5 Mathematica code:

$$
\begin{aligned}
& \text { In[1]:= Simplify }\left[\frac{\pi}{2^{n+1}} \sum_{j=0}^{2^{n}-1} \operatorname{Sin}\left[\frac{(j+1) \pi}{2^{n+1}}\right]\right] \\
& \text { Out[1] }=2^{-2-n} \pi\left(1+\operatorname{Cot}\left[2^{-2-n} \pi\right]\right) \\
& \begin{array}{l}
\operatorname{In}[2]:=\operatorname{Limit}[\%, n \rightarrow \infty] \\
\text { Out[2] }=1
\end{array}
\end{aligned}
$$

we get:

$\sum_{j=0}^{2^{n}-1} \operatorname{vol}\left(\bar{R}_{j}^{n}\right)=\frac{\pi}{2^{n+1}} \sum_{j=0}^{2^{n}-1} \sin \left[\frac{(j+1) \pi}{2^{n+1}}\right]=2^{-2-n} \pi\left(1+\cot \left[2^{-2-n} \pi\right]\right) \rightarrow 1$.

Hence, because $A \subset \bigcup_{j=0}^{2^{n}-1} \bar{R}_{j}^{n}$, we have:

$$
\begin{aligned}
m^{*}(A) & =\inf \left\{\sum_{j=1}^{\infty} \operatorname{vol}\left(R_{j}\right): A \subset \bigcup_{j=1}^{\infty} R_{j}, \quad R_{j} \text { is closed rectangle in } \mathbb{R}^{2}, j \in \mathbb{N}\right\} \\
& \leq \inf \left\{\sum_{j=0}^{2^{n}-1} \operatorname{vol}\left(\bar{R}_{j}^{n}\right): n \in \mathbb{N}\right\} \leq 1 .
\end{aligned}
$$

Step 2.

Using Wolfram Mathematica: 
Listing 6 Mathematica code:

$$
\begin{aligned}
& \text { In[3]:= Simplify }\left[\frac{\pi}{2^{n+1}} \sum_{j=1}^{2^{n}-1} \operatorname{Sin}\left[\frac{j \pi}{2^{n+1}}\right]\right] \\
& \text { Out[3] }=2^{-2-n} \pi\left(-1+\operatorname{Cot}\left[2^{-2-n} \pi\right]\right) \\
& \begin{array}{l}
\operatorname{In}[4]:=\operatorname{Limit}[\%, n \rightarrow \infty] \\
\text { Out[4]= } 1
\end{array}
\end{aligned}
$$

We get that:

$$
\sum_{j=0}^{2^{n}-1} \operatorname{vol}\left(\underline{R}_{j}^{n}\right)=\frac{\pi}{2^{n+1}} \sum_{j=1}^{2^{n}-1} \sin \left[\frac{j \pi}{2^{n+1}}\right]=2^{-2-n} \pi\left(-1+\cot \left[2^{-2-n} \pi\right]\right) \rightarrow 1
$$

Of course, we could use the following formulae: $\sum_{k=1}^{n} \sin (k x)=\frac{\sin \frac{n+1}{2} x \sin \frac{n}{2} x}{\sin \frac{x}{2}}$ and $\lim _{x \rightarrow 0} \frac{\sin x}{x}=1$ instead of the code in Listings 5 and 6 to get the results in formulae (9.1) and (9.3).

We consider an arbitrary covering $A \subset \bigcup_{j=1}^{\infty} R_{j}$ by closed rectangles.

Hence, because $\bigcup_{j=0}^{2^{n}-1} \underline{R}_{j}^{n} \subset A \subset \bigcup_{j=1}^{\infty} R_{j}$ from Lemma 1.5 we have that for any $n$ :

$\sum_{j=0}^{2^{n}-1} \operatorname{vol}\left(\underline{R}_{j}^{n}\right) \leq \sum_{j=1}^{\infty} \operatorname{vol}\left(R_{j}\right)$.

Applying the formula 9.3 we have: $\sum_{j=1}^{\infty} \operatorname{vol}\left(R_{j}\right) \geq \lim _{n \rightarrow \infty} \sum_{j=0}^{2^{n}-1} \operatorname{vol}\left(\underline{R}_{j}^{n}\right)=1$. Consequently,

$m^{*}(A)=\inf \left\{\sum_{j=1}^{\infty} \operatorname{vol}\left(R_{j}\right): A \subset \bigcup_{j=1}^{\infty} R_{j}, \quad R_{j}\right.$ is closed rectangle in $\left.\mathbb{R}^{2}, j \in \mathbb{N}\right\} \geq 1$

From inequalities (9.2) and (9.4) we have $m^{*}(A)=1$ directly from formula (1.3) from Definition 1 . The set $A$ is Lebesgue measurable because $A$ is closed, so $m(A)=1$.

10 Example 9: $A=\left\{(x, y) \in \mathbb{R}^{2}: 0 \leq y \leq \exp x, 0 \leq x \leq 1\right\}$

Let $A$ be the set in $\mathbb{R}^{2}$ bounded by curves: $y=\exp x, y=0$ for $x \in[0,1]$, which means that $A=\{(x, y) \in$ $\left.\mathbb{R}^{2}: 0 \leq y \leq \exp x, 0 \leq x \leq 1\right\}$. Let us calculate Lebesgue outer measure of $A$ using only formula (1.3) from Definition 1 and Lemma 1.5.

For $n \in \mathbb{N}$ define: $\bar{R}_{j}^{n}=\left[\frac{j}{2^{n}}, \frac{j+1}{2^{n}}\right] \times\left[0, \exp \frac{j+1}{2^{n}}\right], j=0,1,2, \ldots 2^{n}-1$ and $\underline{R}_{j}^{n}=\left[\frac{j}{2^{n}}, \frac{j+1}{2^{n}}\right] \times\left[0, \exp \frac{j}{2^{n}}\right]$, $j=0,1,2, \ldots 2^{n}-1$. 
Step 1. Using Wolfram Mathematica:

Listing 7 Mathematica code:

$$
\begin{aligned}
& \operatorname{In}[5]:=\operatorname{Simplify}\left[\frac{1}{2^{n}} \sum_{j=0}^{2^{n}-1} \operatorname{Exp}\left[\frac{(j+1)}{2^{n}}\right]\right] \\
& \text { Out[5] }=\frac{2^{-n}(-1+e) e^{2^{-n}}}{-1+e^{2^{-n}}} \\
& \operatorname{In}[6]:=\operatorname{Limit}[\%, n \rightarrow \infty] \\
& \text { Out[6]= } n+e
\end{aligned}
$$

we get:

$$
\sum_{j=0}^{2^{n}-1} \operatorname{vol}\left(\bar{R}_{j}^{n}\right)=\sum_{j=0}^{2^{n}-1} \exp \frac{(j+1)}{2^{n}} \cdot \frac{1}{2^{n}}=2^{-n}(1-e) e^{-2^{-n}} /\left(-1+e^{2^{-n}}\right) \rightarrow e-1
$$

Hence, because $A \subset \bigcup_{j=0}^{2^{n}-1} \bar{R}_{j}^{n}$, we have:

$$
\begin{aligned}
m^{*}(A) & =\inf \left\{\sum_{j=1}^{\infty} \operatorname{vol}\left(R_{j}\right): A \subset \bigcup_{j=1}^{\infty} R_{j}, \quad R_{j} \text { is closed rectangle in } \mathbb{R}^{2}, j \in \mathbb{N}\right\} \\
& \leq \inf \left\{\sum_{j=0}^{2^{n}-1} \operatorname{vol}\left(\bar{R}_{j}^{n}\right): n \in \mathbb{N}\right\} \leq e-1 .
\end{aligned}
$$

Step 2. Using Wolfram Mathematica:

Listing 8 Mathematica code:

$$
\begin{aligned}
& \operatorname{In}[7]:=\operatorname{Simplify}\left[\frac{1}{2^{n}} \sum_{j=0}^{2^{n}-1} \operatorname{Exp}\left[\frac{j}{2^{n}}\right]\right] \\
& \text { Out[7] }=\frac{2^{-n}(-1+e)}{-1+e^{2^{-n}}} \\
& \operatorname{In}[8]:=\operatorname{Limit}[\%, n \rightarrow \infty] \\
& \text { Out[8]= }-1+e
\end{aligned}
$$

We get that:

$$
\sum_{j=0}^{2^{n}-1} \operatorname{vol}\left(\underline{R}_{j}^{n}\right)=\frac{1}{2^{n}} \sum_{j=0}^{2^{n}-1} \exp \left[\frac{j}{2^{n}}\right]=\frac{2^{-n}(-1+e)}{-1+e^{2^{-n}}} \rightarrow e-1
$$

Of course, we could use the following formulae: $\sum_{k=0}^{n} q^{k}=\frac{1-q^{n+1}}{1-q}(q \neq 1)$ and $\lim _{x \rightarrow 0} \frac{\exp x-1}{x}=1$ instead of the code in Listings 7 and 8 to get the results in formulae (10.1) and (10.3). 
We consider an arbitrary covering $A \subset \bigcup_{j=1}^{\infty} R_{j}$ by closed rectangles.

Hence, because $\bigcup_{j=0}^{2^{n}-1} \underline{R}_{j}^{n} \subset A \subset \bigcup_{j=1}^{\infty} R_{j}$ from Lemma 1.5 we have that for any $n$ :

$\sum_{j=1}^{n} \operatorname{vol}\left(\underline{R}_{j}^{n}\right) \leq \sum_{j=1}^{\infty} \operatorname{vol}\left(R_{j}\right)$.

Applying the formula 10.3 we have: $\sum_{j=1}^{\infty} \operatorname{vol}\left(R_{j}\right) \geq \lim _{n \rightarrow \infty} \sum_{j=1}^{n} \operatorname{vol}\left(\underline{R}_{j}^{n}\right)=1$. Consequently,

$m^{*}(A)=\inf \left\{\sum_{j=1}^{\infty} \operatorname{vol}\left(R_{j}\right): A \subset \bigcup_{j=1}^{\infty} R_{j}, \quad R_{j}\right.$ is closed rectangle in $\left.\mathbb{R}^{2}, j \in \mathbb{N}\right\} \geq e-1$.

From inequalities (10.2) and (10.4) we have $m^{*}(A)=e-1$ directly from formula (1.3) from Definition 1 . The set $A$ is Lebesgue measurable because $A$ is closed, so $m(A)=e-1$.

11 Example 10: $A=\left\{(x, y) \in \mathbb{R}^{2}: 0 \leq y \leq \ln \left(1-2 r \cos x+r^{2}\right), 0 \leq x \leq \pi\right\}(r>1)$

Let $A$ be the set in $\mathbb{R}^{2}$ bounded by curves: $y=\ln \left(1-2 r \cos x+r^{2}\right), y=0$ for $x \in[0, \pi]$, which means that $A=\left\{(x, y) \in \mathbb{R}^{2}: 0 \leq y \leq \ln \left(1-2 r \cos x+r^{2}\right), 0 \leq x \leq \pi\right\}(r>1)$.

Let us calculate Lebesgue outer measure of $A$ using only formula (1.3) from Definition 1 and Lemma 1.5.

Let $f(x)=\ln \left(1-2 r \cos x+r^{2}\right)$.

For $n \in \mathbb{N}$ define: $\bar{R}_{j}^{n}=\left[\frac{j \pi}{2^{n}}, \frac{(j+1) \pi}{2^{n}}\right] \times\left[0, f\left(\frac{(j+1) \pi}{2^{n}}\right)\right], j=0,1,2, \ldots, 2^{n}-1$ and $\underline{R}_{j}^{n}=\left[\frac{j \pi}{2^{n}}, \frac{(j+1) \pi}{2^{n}}\right] \times$ $\left[0, f\left(\frac{j \pi}{2^{n}}\right)\right], j=0,1,2, \ldots, 2^{n}-1$.

Step 1.

In the Mathematica code below we use the fact that if $s_{k}$ is a sequence of positive values with convergent sum, then we have $\sum_{k} s_{k}=\ln \left(\exp \left(\sum_{k} s_{k}\right)\right)=\ln \left(\prod_{k} \exp \left(s_{k}\right)\right)$.

Using Wolfram Mathematica we get:

Listing 9 Mathematica code:

$$
\begin{aligned}
& \operatorname{In}[1]:=g\left[\mathrm{X}_{-}\right]=1-2 r \operatorname{Cos}[\mathrm{x}]+r^{2} ; \\
& \operatorname{In}[2]:=\operatorname{pr}=\operatorname{Simplify}\left[\prod_{k=0}^{n-1} g\left[\frac{k \pi}{n}\right] / \cdot\left\{n \rightarrow 2^{n}\right\}\right] \\
& \text { Out[2]= } \frac{(-1+r)\left(-1+r^{2^{1+n}}\right)}{1+r} \\
& \text { In[3]:= pr1=Simplify }\left[\frac{\mathrm{pr} * g[\pi]}{g[0]}\right] \\
& \text { Out[3] }=\frac{(1+r)\left(-1+r^{2^{1+n}}\right)}{-1+r} \\
& \operatorname{In}[4]:=d=r^{2^{n+1}} ; \\
& \operatorname{In}[5]:=\operatorname{Limit}\left[\frac{\pi}{2^{n}} \log \left[\frac{\mathrm{pr} 1}{d}\right], n \rightarrow \infty, \text { Assumptions } \rightarrow r>1\right]+\operatorname{Limit}\left[\frac{\pi}{2^{n}} \log [d], n \rightarrow \infty, \text { Assumptions } \rightarrow r>1\right] \\
& \text { Out[5] }=2 \pi \log [r]
\end{aligned}
$$

we get:

$$
\sum_{j=0}^{2^{n}-1} \operatorname{vol}\left(\bar{R}_{j}^{n}\right)=\sum_{j=0}^{2^{n}-1} f\left(\frac{(j+1) \pi}{2^{n+1}}\right) \cdot \frac{1}{2^{n}} \pi \rightarrow 2 \pi \ln (r) .
$$


Hence, because $A \subset \bigcup_{j=0}^{2^{n}-1} \bar{R}_{j}^{n}$, we have:

$$
\begin{aligned}
m^{*}(A) & =\inf \left\{\sum_{j=1}^{\infty} \operatorname{vol}\left(R_{j}\right): A \subset \bigcup_{j=1}^{\infty} R_{j}, \quad R_{j} \text { is closed rectangle in } \mathbb{R}^{2}, j \in \mathbb{N}\right\} \\
& \leq \inf \left\{\sum_{j=0}^{2^{n}-1} \operatorname{vol}\left(\bar{R}_{j}^{n}\right): n \in \mathbb{N}\right\} \leq 2 \pi \ln (r) .
\end{aligned}
$$

Step 2. Using Wolfram Mathematica:

Listing 10 Mathematica code:

$$
\begin{aligned}
& \operatorname{In}[6]:=\operatorname{Limit}\left[\frac{\pi}{2^{n}} \log \left[\frac{\mathrm{pr}}{d}\right], n \rightarrow \infty, \text { Assumptions } \rightarrow r>1\right]+\operatorname{Limit}\left[\frac{\pi}{2^{n}} \log [d], n \rightarrow \infty, \operatorname{Assumptions} \rightarrow r>1\right] \\
& \text { Out[6] }=2 \pi \log [r]
\end{aligned}
$$

We get that:

$$
\sum_{j=0}^{2^{n}-1} \operatorname{vol}\left(\underline{R}_{j}^{n}\right)=\sum_{j=0}^{2^{n}-1} f\left(\frac{j \pi}{2^{n+1}}\right) \cdot \frac{1}{2^{n}} \pi \rightarrow 2 \pi \ln (r)
$$

In Listings 9, 10 we used the substitution rule $\left(n \rightarrow 2^{n}\right)$ because when we used directly $2^{n}$ instead $n$, Mathematica could not simplify the expression. We cannot calculate these limits in one step using Mathematica. But using other CAS (wxMaxima, MuPAD) we cannot calculate these limits even in two steps in any way.

Of course, we could use the following formulae (see [3]:

$z^{2 n}-1=\left(z^{2}-1\right) \prod_{k=1}^{n-1}\left(1-2 z \cos (k \pi / n)+z^{2}\right)$

instead of the code in Listings 9 and 10 to get the results in formulae (11.1) and (11.3).

We consider an arbitrary covering $A \subset \bigcup_{j=1}^{\infty} R_{j}$ by closed rectangles.

Hence, because $\bigcup_{j=0}^{2^{n}-1} \underline{R}_{j}^{n} \subset A \subset \bigcup_{j=1}^{\infty} R_{j}$ from Lemma 1.5 we have that for any $n$ :

$\sum_{j=0}^{2^{n}-1} \operatorname{vol}\left(\underline{R}_{j}^{n}\right) \leq \sum_{j=1}^{\infty} \operatorname{vol}\left(R_{j}\right)$.

Applying the formula 11.3 we have: $\sum_{j=1}^{\infty} \operatorname{vol}\left(R_{j}\right) \geq \lim _{n \rightarrow \infty} \sum_{j=0}^{2^{n}-1} \operatorname{vol}\left(\underline{R}_{j}^{n}\right)=2 \pi \ln (r)$. Consequently,

$m^{*}(A)=\inf \left\{\sum_{j=1}^{\infty} \operatorname{vol}\left(R_{j}\right): A \subset \bigcup_{j=1}^{\infty} R_{j}, \quad R_{j}\right.$ is closed rectangle in $\left.\mathbb{R}^{2}, j \in \mathbb{N}\right\} \geq 2 \pi \ln (r)$

From inequalities (11.2) and (11.4) we have $m^{*}(A)=2 \pi \ln (r)$ directly from formula (1.3) from Definition 1 . The set $A$ is Lebesgue measurable because $A$ is closed, so $m(A)=2 \pi \ln (r)$.

12 Example 11: $A=\left\{(x, y) \in \mathbb{R}^{2}: 0 \leq y \leq x^{m}, 0 \leq x \leq 1\right\}(m \in \mathbb{N})$

Let $A$ be the set in $\mathbb{R}^{2}$ bounded by curve and lines: $y=x^{m}, y=0, x=1$ for $x \in[0,1]$, which means that $A=\left\{(x, y) \in \mathbb{R}^{2}: 0 \leq y \leq x^{m}, 0 \leq x \leq 1\right\}$. Let us calculate Lebesgue outer measure of $A$ using only formula (1.3) from Definition 1 and Lemma 1.5. 
For $n \in \mathbb{N}$ define: $\bar{R}_{j}^{n}=\left[\frac{j}{2^{n}}, \frac{j+1}{2^{n}}\right] \times\left[0,\left(\frac{j+1}{2^{n}}\right)^{m}\right], j=0,1,2, \ldots 2^{n}-1$ and $\underline{R}_{j}^{n}=\left[\frac{j}{2^{n}}, \frac{j+1}{2^{n}}\right] \times\left[0,\left(\frac{j}{2^{n}}\right)^{m}\right]$, $j=0,1,2, \ldots 2^{n}-1$.

Step 1. Using Wolfram Mathematica:

Listing 11 Mathematica code:

$$
\begin{aligned}
& \operatorname{In}[1]:=\operatorname{Limit}\left[\frac{1}{2^{n}} \sum_{j=0}^{2^{n}-1}\left(\frac{j+1}{2^{n}}\right)^{m}, n \rightarrow \infty, \text { Assumptions } \rightarrow m \in \text { Integers } \& \& m>0\right] \\
& \text { Out }[1]=\frac{1}{1+m}
\end{aligned}
$$

we get: $\sum_{j=0}^{2^{n}-1} \operatorname{vol}\left(\bar{R}_{j}^{n}\right)=\sum_{j=0}^{2^{n}-1}\left(\frac{j+1}{2^{n}}\right)^{m} \cdot \frac{1}{2^{n}} \rightarrow \frac{1}{m+1}$.

Hence, because $A \subset \bigcup_{j=0}^{2^{n}-1} \bar{R}_{j}^{n}$, we have:

$$
\begin{aligned}
m^{*}(A) & =\inf \left\{\sum_{j=1}^{\infty} \operatorname{vol}\left(R_{j}\right): A \subset \bigcup_{j=1}^{\infty} R_{j}, \quad R_{j} \text { is closed rectangle in } \mathbb{R}^{2}, j \in \mathbb{N}\right\} \\
& \leq \inf \left\{\sum_{j=0}^{2^{n}-1} \operatorname{vol}\left(\bar{R}_{j}^{n}\right): n \in \mathbb{N}\right\} \leq \frac{1}{m+1} .
\end{aligned}
$$

Step 2.

Using Wolfram Mathematica:

Listing 12 Mathematica code:

$$
\begin{aligned}
& \operatorname{In}[2]:=\operatorname{Limit}\left[\frac{1}{2^{n}} \sum_{j=0}^{2^{n}-1}\left(\frac{j}{2^{n}}\right)^{m}, n \rightarrow \infty, \text { Assumptions } \rightarrow m \in \operatorname{Integers} \& \& m>0\right] \\
& \text { Out[2] }=\frac{1}{1+m}
\end{aligned}
$$

We get that:

$$
\sum_{j=0}^{2^{n}-1} \operatorname{vol}\left(\underline{R}_{j}^{n}\right)=\sum_{j=0}^{2^{n}-1}\left(\frac{j}{2^{n}}\right)^{m} \cdot \frac{1}{2^{n}} \rightarrow \frac{1}{m+1} .
$$

Of course, we could use the Stolz and binomial theorems instead of the code in Listings 11 and 12 to get the results in formulae (12.1) and (12.2).

We consider an arbitrary covering $A \subset \bigcup_{j=1}^{\infty} R_{j}$ by closed rectangles.

Hence, because $\bigcup_{j=0}^{2^{n}-1} \underline{R}_{j}^{n} \subset A \subset \bigcup_{j=1}^{\infty} R_{j}$ from Lemma 1.5 we have that for any $n$ :

$\sum_{j=0}^{2^{n}-1} \operatorname{vol}\left(\underline{R}_{j}^{n}\right) \leq \sum_{j=1}^{\infty} \operatorname{vol}\left(R_{j}\right)$. 
Applying the formula 12.2 we have: $\sum_{j=1}^{\infty} \operatorname{vol}\left(R_{j}\right) \geq \lim _{n \rightarrow \infty} \sum_{j=0}^{2^{n}=1} \operatorname{vol}\left(\underline{R}_{j}^{n}\right)=\frac{1}{m+1}$. Consequently,

$m^{*}(A)=\inf \left\{\sum_{j=1}^{\infty} \operatorname{vol}\left(R_{j}\right): A \subset \bigcup_{j=1}^{\infty} R_{j}, \quad R_{j}\right.$ is closed rectangle in $\left.\mathbb{R}^{2}, j \in \mathbb{N}\right\} \geq \frac{1}{m+1}$.

From inequalities (12.1) and (12.3) we have $m^{*}(A)=\frac{1}{m+1}$ directly from formula (1.3) from Definition 1. The set $A$ is Lebesgue measurable because $A$ is closed, so $m(A)=\frac{1}{m+1}$.

\section{Conclusions}

In this paper the authors presented several examples of Lebesgue outer measure calculated directly from its definition using Mathematica.

We could not find any analogical examples in available literature, so this paper is an attempt to fill this gap.

Using Mathematica or other CAS programs for calculation Lebesgue outer measure directly from its definitions, seems to be didactically useful for students because of the possibility of symbolic calculation of sums, limits and plot graphs - checking our hand calculations. Moreover, we get students used not only to definition of Lebesgue outer measure but also to CAS applications generally.

Open Access This article is distributed under the terms of the Creative Commons Attribution 4.0 International License (http:// creativecommons.org/licenses/by/4.0/), which permits unrestricted use, distribution, and reproduction in any medium, provided you give appropriate credit to the original author(s) and the source, provide a link to the Creative Commons license, and indicate if changes were made.

\section{References}

1. Aliprantis, C.D., Burkinshaw, O.: Principles of Real Analysis, 2nd edn. Academic Press, Cambridge (1991)

2. Bartle, R.G.: The Elements of Integration and Lebesgue Measure. Wiley-Interscience, Hoboken (1995)

3. Fichtenholz, G.M.: Differential and Integral Calculus, 3rd edn. Fizmatgiz, Moscow (1958)

4. Folland, G.B.: Real Analysis Modern Technique, 2nd edn. Wiley, Hoboken (2007)

5. Jones, F.: Lebesgue Integration on Euclidean Space. Jones \& Bartlett Learning, Burlington (2000)

6. Kołodziej, W.: Mathematical Analysis. Polish Scientific Publishers PWN, Warsaw (2012). (in polish)

7. Ruskeepa, H.: Mathematica Navigator: Graphics and Methods of applied Mathematics. Academic Press, Boston (2005)

8. Sikorski, R.: Differential and Integral Calculus. Functions of Several Variables, 2nd edn. Polish Scientific Publishers PWN, Warsaw (1977). (in polish)

9. Stein, E.M., Shakarchi, R.: Real Analysis: Measure Theory, Integration, and Hilbert Spaces. Princeton University Press, Princeton (2003)

10. Stroock, D.W.: A Concise Introduction to the Theory of Integration, 3rd edn. Birkhäuser, Basel (1998)

11. Wojas, W., Krupa, J.: Familiarizing students with definition of lebesgue integral: examples of calculation directly from its definition using mathematica. Math. Comput. Sci. 11, 363-381 (2017)

12. Wolfram, S.: The Mathematica Book. Wolfram Media Cambridge University Press, Cambridge (1996)

Publisher's Note Springer Nature remains neutral with regard to jurisdictional claims in published maps and institutional affiliations. 